\title{
Principle component analysis of myocardial strain to quantify left ventricular dyssynchrony
}

\author{
Raghav Ramachandran ${ }^{1 *}$, Xiao Chen ${ }^{1}$, Bhairav B Mehta ${ }^{1}$, Kenneth C Bilchick ${ }^{2}$, Frederick H Epstein ${ }^{1}$ \\ From 16th Annual SCMR Scientific Sessions \\ San Francisco, CA, USA. 31 January - 3 February 2013
}

\begin{abstract}
Background
Cardiac resynchronization therapy (CRT) is effective for selected heart failure (HF) patients, but is associated with a $30-40 \%$ nonresponse rate. Identification of CRT responders may be improved with myocardial strain imaging. The circumferential uniformity ratio estimate $(\text { CURE })^{1}$ measures mechanical dyssynchrony by Fourier series fitting of myocardial strains over space, but requires user interaction to define a range of cardiac phases over which CURE is calculated (time dependence). We hypothesize that principal component analysis (PCA) can quantify dyssynchrony in myocardial strain in a data-driven, time-independent manner that does not require any subjective user assessments of strain data.
\end{abstract}

\section{Methods}

Dyssynchronous HF was induced in canines $(\mathrm{N}=5)$ with tachycardia pacing and left bundle branch ablation (LBBB-HF), while synchronous HF with narrow QRS (NQRS-HF) was induced in canines with tachycardia pacing only $(\mathrm{N}=5)$. Four normal canines were also studied.

Spiral cine DENSE MRI was performed on a $1.5 \mathrm{~T}$ scanner (Avanto, Siemens) in all 14 canines. 2D myocardial motion was tracked in a mid-ventricular short-axis plane from DENSE images acquired using the following parameters $^{2}$ : interleaves $=6, \mathrm{TR}=17 \mathrm{~ms}, \mathrm{TE}=1.9 \mathrm{~ms}$, slice thickness $=8 \mathrm{~mm}$, excitation flip angle $=15^{\circ}$, in-plane resolution $=2.8 \times 2.8 \mathrm{~mm}$ and displacement-encoding frequency $=0.1$ cycles $/ \mathrm{mm}$.

DENSE images were analyzed ${ }^{3}$ to calculate left ventricular (LV) circumferential strain (Ecc), and PCA was applied to the Ecc-time curves for a 24-segment LV model. Specifically, the LV Ecc curves were decomposed spatially into principal component basis vectors. The PCA-based metric for measuring LV dyssynchrony, termed First Order Regional Conformity Estimate (FORCE), was calculated as $\left|\operatorname{sum}\left(\mathrm{PCL}_{1}\right)\right| / \operatorname{sum}\left(\left|\mathrm{PCL}_{1}\right|\right)$ where $\mathrm{PCL}_{1}$ represents the loadings of the first principal component basis vector. Both FORCE and CURE range from 0 (dyssynchrony) to 1 (perfect synchrony). The Kruskal-Wallis one-way ANOVA test was used to compare FORCE and CURE among the three groups in pairwise fashion.

\section{Results}

Figure 1 shows the spatial distribution of $\mathrm{PCL}_{1}$ for the Ecc of example LBBB-HF and NQRS-HF canines. The $\mathrm{PCL}_{1}$ of LV Ecc in LBBB-HF canines varied widely over $\mathrm{LV}$ segments, whereas $\mathrm{PCL}_{1}$ showed little variation over LV segments in NQRS-HF and normal canines. As shown in Figure 2, FORCE and CURE were both markedly different in LBBB-HF versus NQRS-HF $(\mathrm{p}<0.05)$ and LBBB-HF versus normal $(\mathrm{p}<0.05)$. Also, FORCE was significantly greater than CURE for NQRS-HF $(\mathrm{p}<0.05)$, indicating even better identification of synchrony than CURE.

\section{Conclusions}

PCA, using the promising new FORCE parameter, effectively and automatically identifies mechanical dyssynchrony in HF in a data-driven and completely time-independent fashion. Further clinical evaluation of FORCE for prediction of CRT response is warranted.

\section{Funding}

This work was funded by the American Heart Association 12GRNT12050301 and NIH Heart, Lung and Blood Institute (NHLBI) T32HL007284. 


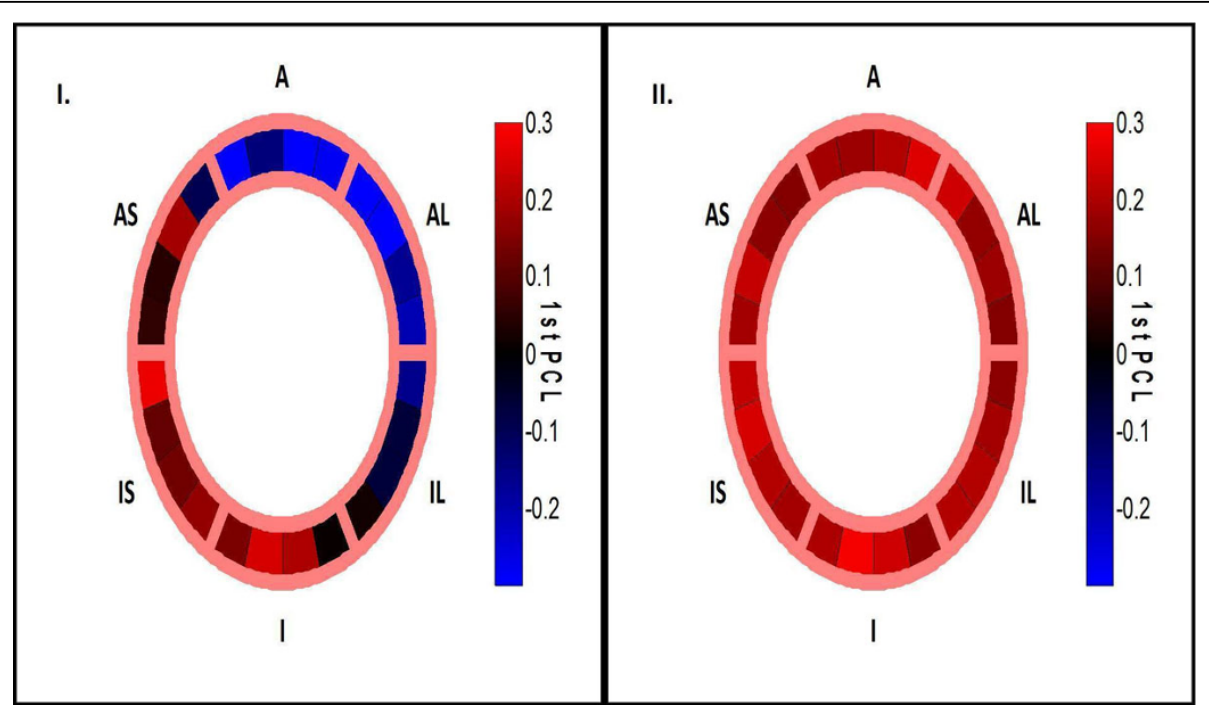

Figure 1 Bulls-eye plots of $\mathrm{PCL}_{1}$ as a function of LV segments for I) a LBBB-HF and II) a NQRS-HF canine; A-Anterior, AL-Anterolateral, ILInferolateral, I-Inferior, IS-Inferoseptal, AS-Anteroseptal.

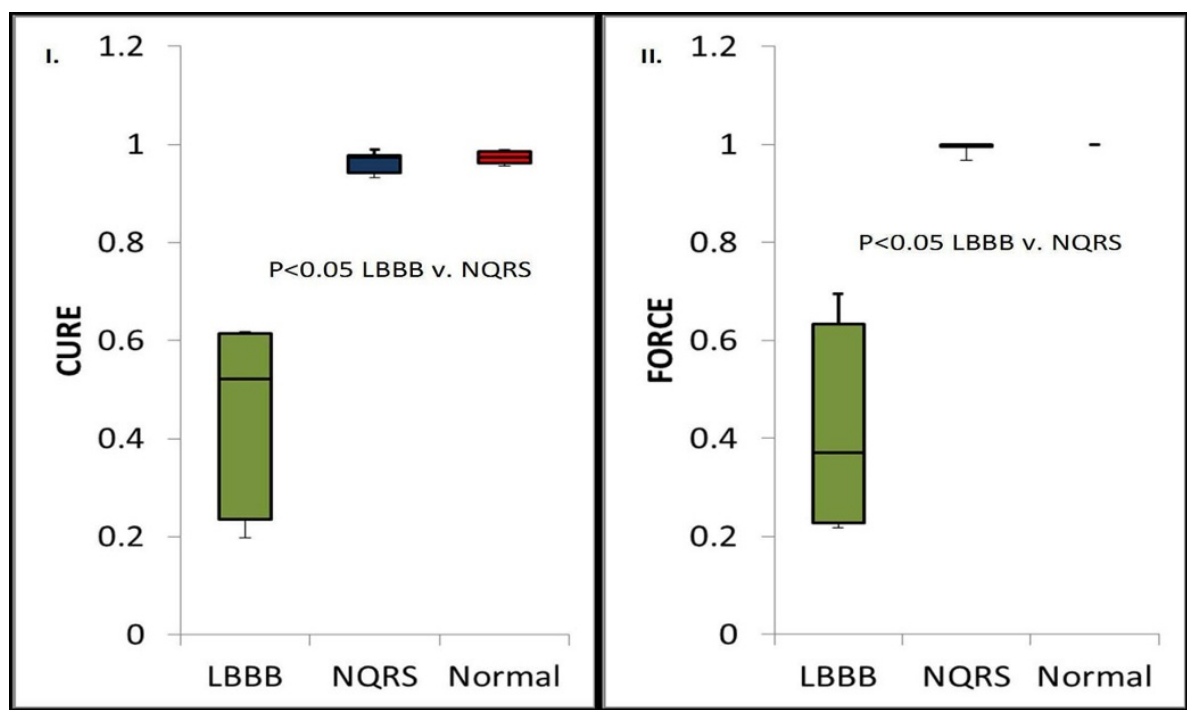

Figure 2 Boxplot comparisons of I) CURE and II) FORCE for LBBB-HF,NQRS-HF and Normal dogs.

\section{Author details}

${ }^{1}$ Biomedical Engineering, University of Virginia, Charlottesville, VA, USA.

${ }^{2}$ Department of Medicine, University of Virginia, Charlottesville, VA, USA.

Published: 30 January 2013

\section{References}

1. Bilchick, et al:. JACC Imaging 2008 .

2. Budge, et al:. JACC Imaging 2012.

3. Spottiswoode, et al:. IEEE Trans Med Imaging 2007.

doi:10.1186/1532-429X-15-S1-P74

Cite this article as: Ramachandran et al:: Principle component analysis of

myocardial strain to quantify left ventricular dyssynchrony. Journal of

Cardiovascular Magnetic Resonance 2013 15(Suppl 1):P74. 